\title{
GATA3 expression in paragangliomas: a pitfall potentially leading to misdiagnosis of urothelial carcinoma
}

\author{
Jeffrey S So ${ }^{1}$ and Jonathan I Epstein ${ }^{1,2,3}$ \\ ${ }^{1}$ Department of Pathology, The Johns Hopkins Medical Institutions, Baltimore, MD, USA; ${ }^{2}$ Department \\ of Urology, The Johns Hopkins Medical Institutions, Baltimore, MD, USA and ${ }^{3}$ Department of Oncology, \\ The Johns Hopkins Medical Institutions, Baltimore, MD, USA
}

\begin{abstract}
GATA3 is a zinc-finger transcription factor, which is expressed in various normal and neoplastic tissues. Amongst tumors, it labels urothelial carcinoma, collecting duct carcinoma of the kidney, breast carcinoma, lymphoma and, uncommonly, endometrial carcinoma. Few studies have investigated its positivity in various neoplasms that may mimic urothelial neoplasms. In this study, we evaluated GATA3 expression in urinary bladder paragangliomas, which may closely mimic urothelial carcinomas. We retrieved 12 cases of paragangliomas from the urinary bladder and 20 cases of paragangliomas from non-urologic sites using the Hopkins Pathology Data Base system. GATA3 was positive in 10 of the $12(83 \%)$ urinary bladder paragangliomas studied on routine slide sections. Most $(6 / 12)$ of the staining was diffusely strong $(3+)$ staining, whereas the rest $(4 / 12)$ that were positive showed mixed intensities (strong $3+$ to moderate $2+$ ). The 20 paragangliomas from other sites were constructed into tissue microarrays, wherein three cores from each tumor were taken. Fifteen out of $20(75 \%)$ paragangliomas outside of the bladder were positive for GATA3 staining. Moderate $(2+)$ or strong $(3+)$ staining was seen in $13 / 20(65 \%)$ of extravesical paragangliomas, ranging from 5 to $100 \%$ of the cell labeling (mean $59 \%$, median $60 \%$ ). In the remaining $7 / 20(35 \%)$ cases, only weak (2/7) or negative (5/7) immunoreactivity for GATA3 was seen. An additional 15 cases of metastatic paraganglioma from various primary sites were retrieved with 12 of $15(80 \%)$ metastatic paragangliomas staining positively for GATA3. Overall, for paragangliomas, regardless of site, $78.7 \%$ were positive for GATA3. Recognition of this finding will aid pathologists in preventing a misdiagnosis of a urothelial tumor based on GATA3 expression, which is critical given the differences in treatment, follow-up and prognosis between bladder paragangliomas and urothelial carcinoma.

Modern Pathology (2013) 26, 1365-1370; doi:10.1038/modpathol.2013.76; published online 19 April 2013
\end{abstract}

Keywords: GATA3; paraganglioma; urothelial carcinoma

GATA3 is one member of the zinc-finger transcription family involved in the regulation, speciation, differentiation and proliferation of cells during development of multiple organs, ${ }^{1-14}$ including the nervous ${ }^{13}$ and urogenital system. ${ }^{3}$ Amongst tumors, it labels urothelial carcinoma, collecting duct carcinoma of the kidney, breast carcinoma, lymphoma and, uncommonly, endometrial carcinoma. Few studies have investigated its positivity in various neoplasms that may mimic urothelial

Correspondence: Dr JI Epstein, MD, Department of Pathology, The Johns Hopkins Medical Institutions, Weinberg 2242, 401 North Broadway, Baltimore, MD 21231, USA.

E-mail: jepstein@jhmi.edu

Received 14 January 2013; revised 7 March 2013;

accepted 7 March 2013; published online 19 April 2013 neoplasms, and we are unaware of prior studies evaluating GATA3 in paragangliomas. In this study, we evaluated GATA3 expression in urinary bladder paragangliomas, which may closely mimic urothelial carcinomas, especially in small biopsy specimens.

\section{Materials and methods}

A search using the Hopkins Pathology Data Base system identified 12 cases (8 consult cases) of paraganglioma from the urinary bladder, where we were able to retrieve paraffin blocks or unstained slides. In addition, 20 cases of paragangliomas from various non-urologic sites (vagus nerve, temporal bone, dura, jugular, carotid body, retroperitoneum, 
skull base, pelvic area, periaortic area and paratracheal area) were identified from the same database and constructed into two tissue microarray paraffin blocks. Three tissue cores were obtained from each tumor for the tissue microarrays. Another 15 cases of paragangliomas from various sites metastatic to the spine $(n=9)$, epidural soft tissue $(n=1)$, mesenteric lymph node $(n=1)$, periaortic lymph node $(n=2)$, liver $(n=1)$ and right paratracheal area $(n=1)$ were retrieved. Immunohistochemical staining was accomplished with the GATA3 antibody (catalogue number L50-823 clone, 1:100 dilution; Biocare Medical, Concord, CA). Staining was performed on a fully automated system (Ventana Medical Systems, Tucson, AZ). Slides were deparaffinized and antigen retrieval was performed with mild cell conditioning CC1 solution ( $30 \mathrm{~min}$ ). Primary antibody incubation was done for $44 \mathrm{~min}$ followed by an amplification step, and the test was developed using Ultra-View polymer detection kit (Ventana Medical Systems) as per the manufacturer's instruction.

The authors did a visual assessment of GATA3 expression over a multiheaded microscope. GATA3 positivity was only considered if it was seen in a nuclear staining pattern. Staining intensity was assigned a score of 0 (negative), $1+$ (weak), $2+$ (moderate) or $3+$ (strong). For each tumor, a percentage was assigned for the number of cells staining with a particular staining intensity.

\section{Results}

Patients' whose tumors were located in the urinary bladder were of 26-85 years old (median 60.5). Paragangliomas located elsewhere occurred in patients with an age range of 4-65 years (median 46).

GATA3 was positive in 10 of the $12(83.3 \%)$ urinary bladder paragangliomas studied on routine slide sections (Figure 1). Six cases $(50 \%)$ had diffusely strong $(3+)$ staining. Four cases $(33 \%)$ showed mixed intensities (strong $3+$ to moderate $2+$ ), whereas the remaining two cases (17\%) were negative. Fifteen of $20(75 \%)$ paragangliomas outside of the bladder were positive for GATA3 staining (Figure 2). Moderate $(2+)$ or strong $(3+)$ staining was seen in 13/20 (65\%) of extravesical paragangliomas, ranging from 5 to $100 \%$ of the cell labeling (mean 58.5\%, median 60\%). In the remaining $7 / 20$ $(35 \%)$ cases, only weak $(2 / 7)$ or negative $(5 / 7)$ immunoreactivity for GATA3 was seen. Twelve of $15(80 \%)$ metastatic paragangliomas stained positively for GATA3 (Tables 1-3).

\section{Discussion}

Of paragangliomas throughout the body, $10 \%$ occur in extra-adrenal sites; and of these extraadrenal tumors, $10 \%$ are found in the urinary bladder wall. ${ }^{15,16}$ Paragangliomas of the urinary bladder account for less than $0.5 \%$ of bladder tumors. Primary paragangliomas of the bladder, which have also been incorrectly designated in the literature as bladder pheochromocytomas, have a female to male ratio of $3: 2$, with an average age diagnosis of 41 years. There is a wide age distribution from childhood to the elderly. Most tumors are located either on the dome or the trigone. Although the majority of patients have exophytic lesions with an intact mucosa, in a minority of cases there may be ulceration of the overlying mucosa. Lesions vary greatly in size ranging up to $10 \mathrm{~cm}$, with the majority of patients presenting with solitary lesions.

Paragangliomas can often be diagnosed on clinical grounds. Seventy-five percent of patients experience micturition attacks. When the bladder fills or upon urination, there ensues bursts of headaches, palpitations, hypertension, blurred vision, sweating, anxiety, tremulousness and sometimes syncope. Hypertension can also be induced by intraoperative manipulation of the tumor. Increases in serum or urine catecholamines can provide laboratory confirmation of the diagnosis. Radiologically, bladder paragangliomas may be diagnosed using arteriography, selective venous sampling, CT or MRI, and scintigraphy with meta- ${ }^{131}$ iodobenzyoguanidine.

Histologically, paragangliomas typically are composed of nests (Zellballen) separated by either a thin delicate plexiform vascular network or more fibrous septa. At higher magnification, polyhedric cells have acidophilic to amphophilic cytoplasm with central or eccentric nuclei and visible nucleoli. Some of the features seen in paragangliomas can cause diagnostic difficulties and be misdiagnosed as urothelial carcinoma. As in many endocrine tumors, occasional bizarre nuclei may be seen in urinary paragangliomas. However, the chromatin appears smudged and hyperchromatic without mitoses, typical of degenerative atypia rather than the atypia of a high-grade malignant tumor. Mitoses in paragangliomas may be present, although typically not numerous. Paragangliomas of the bladder can invade deeply into the muscularis propria or show vascular invasion. Focal hemorrhage and necrosis may also be present. Another histological feature that can lead to a misdiagnosis of urothelial carcinoma is a diffuse growth pattern, with significant cautery artifact compounding the diagnostic problems. ${ }^{17}$ In addition, when pathologists are confronted with an invasive tumor in the bladder at all reminiscent of urothelial carcinoma, the default diagnosis is often urothelial carcinoma, given that it accounts for the vast majority of tumors involving the bladder. Invasive urothelial carcinoma on occasion can also grow in a nesting pattern reminiscent of paraganglioma.

The distinction between paraganglioma and urothelial cancer is critical because of different therapeutic options. Localized paragangliomas are treated by transurethral resection, wedge resection 

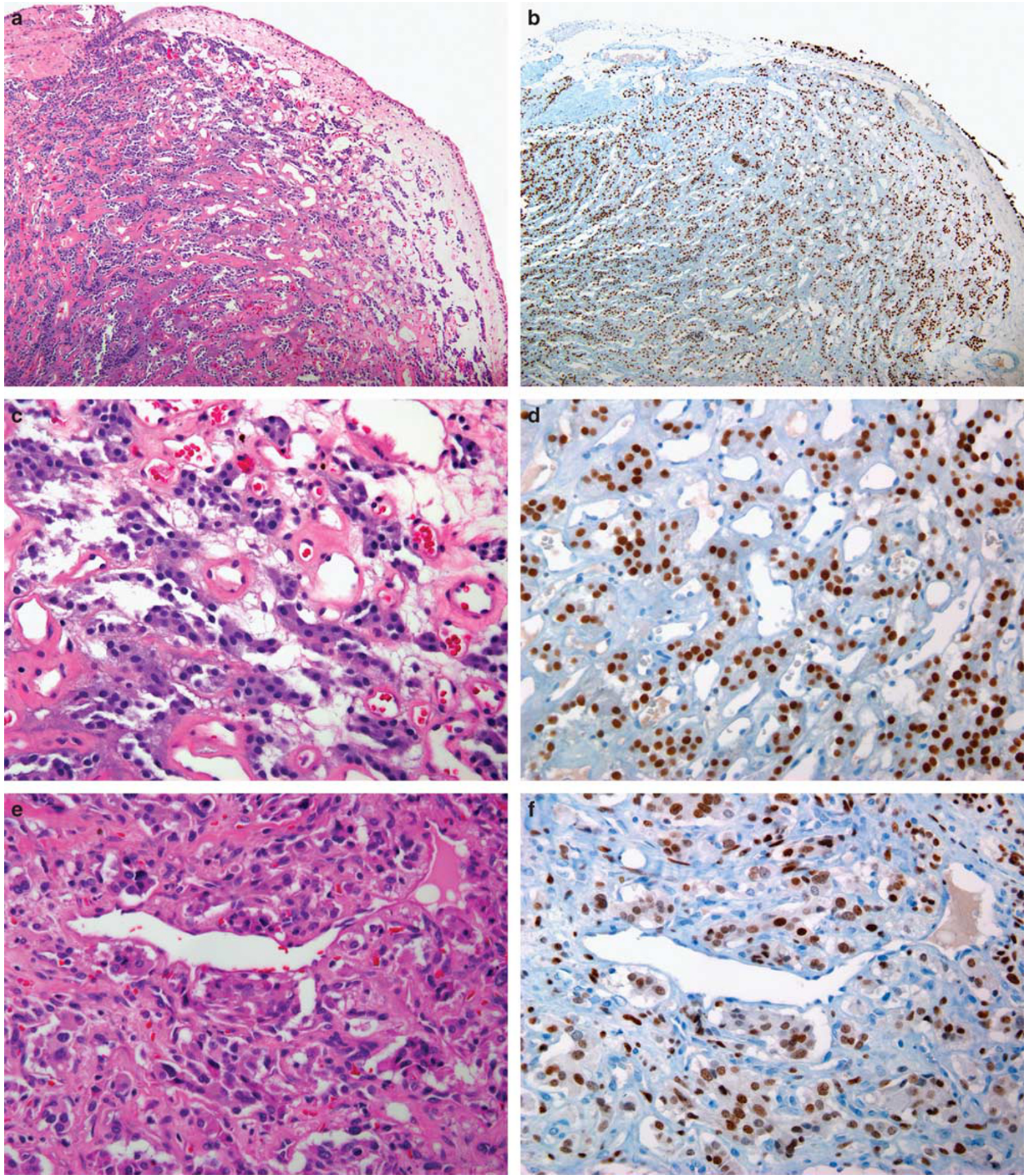

Figure 1 (a) Low magnification of urinary bladder paraganglioma undermining urothelium. (b) Low magnification of diffuse $3+$ GATA3 staining (same case as a). (c) Higher magnification of a showing typical nested morphology of paraganglioma. (d) Higher magnification of b with diffuse $3+$ GATA3 immunoreactivity. (e) Separate case of urinary bladder paraganglioma. (f) Mixture of $3+$ and $2+$ GATA3 immunoreactivity (same case as $\mathbf{e}$ ).

or partial cystectomy. There is no need to follow patients for multifocal disease. There are no histologic criteria (ie, atypia, vascular invasion, size, etc) predictive of which lesions are capable of distant spread. Only those tumors that have metastasized are diagnosed as malignant. Approximately 10-15\% of paragangliomas of the bladder are malignant. Radiation and chemotherapy appear to have limited 

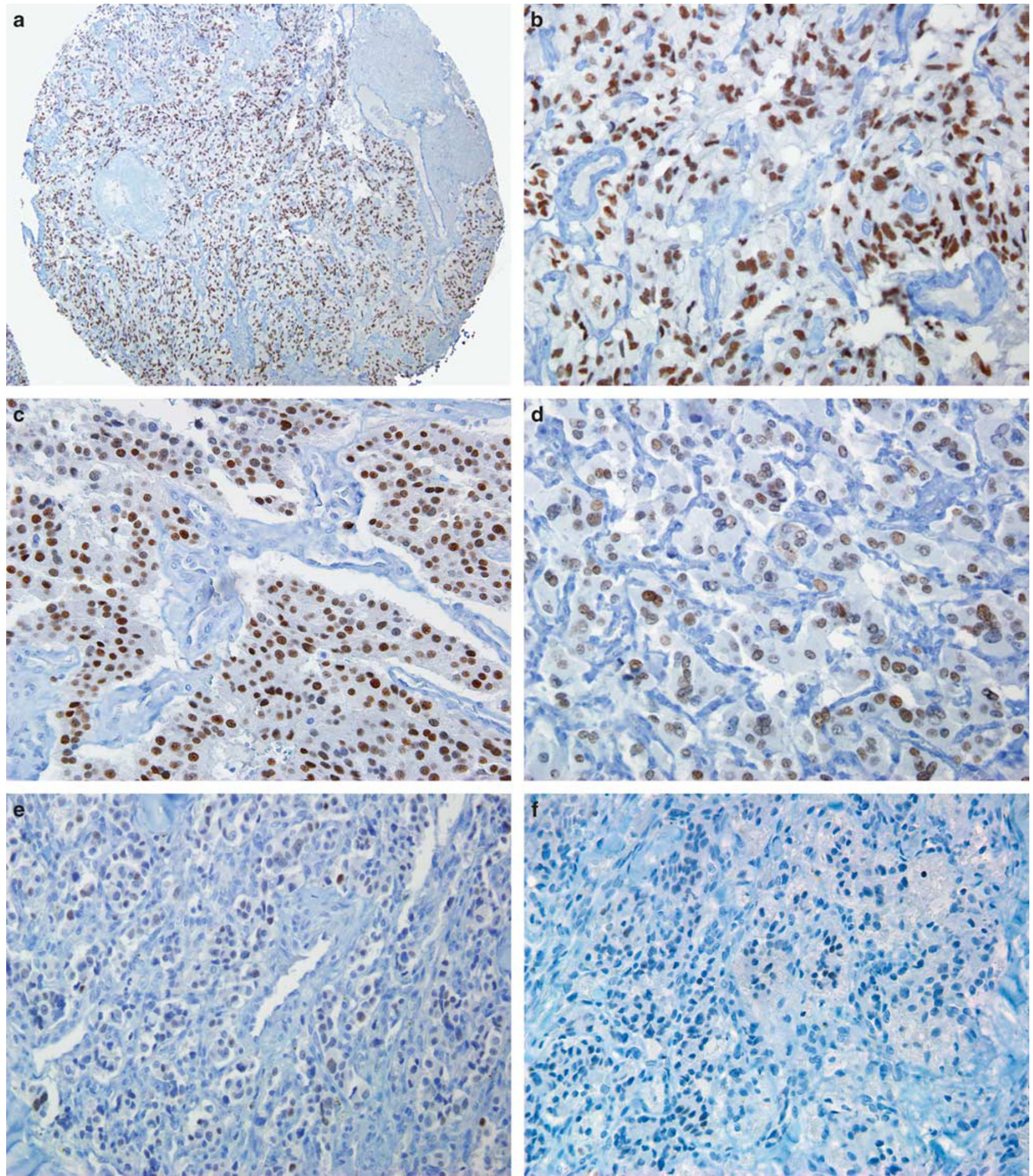

Figure 2 (a, b) Low and high magnification of diffuse $3+$ GATA3 staining in tissue microarray of paraganglioma involving the pelvis. (c) Mixture of $3+$ and $2+$, and $1+$ GATA3 immunoreactivity in retroperitoneal paraganglioma. (d) $2+$ and $1+$ GATA3 staining in soft tissue paraganglioma. (e) Carotid body paraganglioma with $1+$ and negative GATA3 staining. (f) Carotid body paraganglioma with negative GATA3 immunoreactivity.

effectiveness for paragangliomas. In contrast, muscleinvasive urothelial carcinoma is typically treated by radical cystectomy and patients need to be followed for life for upper tract and urethral multifocal disease. Chemotherapy is used for advanced urothelial carcinoma.

If one considers the diagnosis of paraganglioma, immunohistochemistry is helpful in that tumors 
Table 1 Urinary bladder paragangliomas staining intensities

\begin{tabular}{lcccc}
\hline Patient & $3+$ & $2+$ & $1+$ & Negative \\
\hline 1 & Diffuse & & & \\
2 & Diffuse & & & \\
3 & Diffuse & & & \\
4 & Diffuse & & & \\
5 & Diffuse & & & \\
6 & Diffuse & & & \\
7 & & $40 \%$ & $70 \%$ & $100 \%$ \\
8 & & $30 \%$ & & $100 \%$ \\
9 & & $30 \%$ & & \\
10 & & $20 \%$ & & \\
11 & & & & \\
12 & & & & \\
\hline
\end{tabular}

Table 2 Non-Vesical Paragangliomas staining intensities

\begin{tabular}{|c|c|c|c|c|}
\hline Number of cases & \multicolumn{4}{|c|}{ Staining intensity and percentage (\%) } \\
\hline 1 & $3+(100)$ & & & \\
\hline 1 & $3+(80)$ & $2+(10)$ & & Negative (10) \\
\hline 1 & $3+(80)$ & & & Negative (20) \\
\hline 1 & $3+(30)$ & $2+(30)$ & $1+(30)$ & Negative (10) \\
\hline 3 & $3+(10)$ & $2+(80)$ & $1+(10)$ & \\
\hline 1 & & $2+(50)$ & $1+(40)$ & Negative (10) \\
\hline 1 & & $2+(40)$ & $1+(30)$ & Negative (30) \\
\hline 2 & & $2+(30)$ & $1+(30)$ & Negative (40) \\
\hline 2 & & $2+(5)$ & & Negative (95) \\
\hline 1 & & & $1+(80)$ & Negative (20) \\
\hline 1 & & & $1+(20)$ & Negative (80) \\
\hline 5 & & & & Negative (100) \\
\hline
\end{tabular}

Table 3 Metastatic Paragangliomas Staining Intensities

\begin{tabular}{lllll}
\hline \multirow{2}{*}{ Number of cases } & \multicolumn{5}{c}{ Staining intensities (\%) } \\
\hline 1 & $3+(60)$ & $2+(40)$ & & $0(10)$ \\
2 & $3+(50)$ & $2+(40)$ & & \\
1 & $3+(40)$ & $2+(40)$ & $1+(20)$ & \\
1 & $3+(30)$ & $2+(40)$ & $1+(30)$ & \\
2 & $3+(20)$ & $2+(30)$ & $1+(30)$ & $0(20)$ \\
1 & $3+(20)$ & $2+(20)$ & $1+(10)$ & $0(50)$ \\
1 & $3+(10)$ & $2+(80)$ & $1+(10)$ & \\
1 & $3+(10)$ & $2+(30)$ & $1+(10)$ & $0(50)$ \\
1 & & $2+(20)$ & $1+(50)$ & $0(30)$ \\
1 & & & $1+(10)$ & $0(90)$ \\
3 & & & & $0(100)$ \\
& & & &
\end{tabular}

usually react with antisera to synaptophysin, chromogranin and S100 (sustentacular cells surrounding tumor nests), with negative results for cytokeratin. However, if one does not think of paraganglioma and uses a marker, such as GATA3, to demonstrate urothelial differentiation, the current study illustrates the potential of GATA3 positivity in paragangliomas to lead to a misdiagnosis.

Two large studies have reported that GATA3 has a sensitivity of 67 and $87 \%$ for urothelial carcinoma. ${ }^{18,19}$ The only other carcinomas with GATA3 positivity that have been reported are carcinomas of the breast (ductal 91\%, lobular $100 \%$ ) and rarely endometrial carcinoma. ${ }^{2}$ GATA3 is currently, most commonly used in the bladder to differentiate between urothelial carcinoma and high-grade prostatic adenocarcinoma. ${ }^{20}$ Whereas there are sensitive and specific markers for prostate adenocarcinoma, such as PSA, p501S and NKX 3.1, before GATA3, there were no sensitive and specific immunohistochemical antibodies for urothelial carcinoma. Uroplakin is specific for urothelial carcinoma, yet not sensitive in highgrade tumors. High molecular weight cytokeratin and p63 have high specificity and increased sensitivity in terms of differentiating urothelial from prostate carcinoma, yet their sensitivity is still only $60-70 \%$ and is not specific for urothelial carcinoma in other settings.

Whether in the bladder assessing a primary tumor, or in other sites evaluating for possible metastatic carcinoma coming from the urinary bladder, GATA3 immunoreactivity in the vast majority of cases is associated with a urothelial carcinoma. However, the current study demonstrates that in rare cases, GATA3 positivity may indicate a paraganglioma, which should be evaluated with additional immunohistochemical stains along with clinical history and morphology.

\section{Disclosure/conflict of interest}

The authors declare no conflict of interest.

\section{References}

1 Hosoya T, Maillard I, Engel JD. From the cradle to the grave: activities of GATA-3 throughout $\mathrm{T}$ cell development and differentiation. Immunol Rev 2010; 238:110-125.

2 Asselin-Labat ML, Sutherland KD, Barker $\mathrm{H}$, et al. Gata-3 is an essential regulator of mammary-gland morphogenesis and luminal-cell differentiation. Nat Cell Biol 2006;9:201-209.

3 Grote D, Souabni A, Busslinger M, et al. Pax2/8regulated Gata3 expression is necessary for morphogenesis and guidance of the nephric duct in the developing kidney. Development 2006;133:53-61.

4 Kouros-Mehr H, Slorach EM, Sternlicht MD, et al. GATA-3 maintains the differentiation of the luminal cell fate in the mammary gland. Cell 2006;127: 1041-1055.

5 Burch JB. Regulation of GATA gene expression during vertebrate development. Semin Cell Dev Biol 2005; 16:71-81.

6 Zheng R, Blobel GA. GATA transcription factors and cancer. Genes Cancer 2010;1:1178-1188.

7 Yoon NK, Maresh EL, Shen D, et al. Higher levels of GATA3 predict better survival in women with breast cancer. Hum Pathol 2010;41:1794-1801.

8 Ting CN, Olson MC, Barton KP, et al. Transcription factor GATA-3 is required for development of the T-cell lineage. Nature 1996;384:474-478. 
9 Naito T, Tanaka H, Naoe Y, et al. Transcriptional control of T-cell development. Int Immunol 2011; 23:661-668.

10 Pai SY, Truitt ML, Ting CN, et al. Critical roles for transcription factor GATA-3 in thymocyte development. Immunity 2003;19:863-875.

11 Hendriks RW, Nawijn MC, Engel JD, et al. Expression of the transcription factor GATA-3 is required for the development of the earliest $\mathrm{T}$ cell progenitors and correlates with stages of cellular proliferation in the thymus. Eur J Immunol 1999;29:1912-1918.

12 Tong Q, Dalgin G, Xu H, et al. Function of GATA transcription factors in preadipocyte-adipocyte transition. Science 2000;290:134-138.

13 Tsarovina K, Pattyn A, Stubbusch J, et al. Essential role of Gata transcription factors in sympathetic neuron development. Development 2004;131:4775-4786.

14 Kaufman CK, Zhou P, Pasolli HA, et al. GATA-3:an unexpected regulator of cell lineage determination in skin. Genes Dev 2003;17:2108-2122.

15 Cheng L, Leibovich BC, Cheville JC, et al. Paraganglioma of the urinary bladder: can biologic potential be predicted? Cancer 2000;88:844-852.
16 Naktani T, Hayama T, Uchida J, et al. Diagnostic localization of extra-adrenal pheochromocytoma: comparison of (123)I-MIBG imaging and (131)I-MIBG imaging. Oncol Rep 2002;9:1225-1227.

17 Zhou M, Epstein JI, Young R. Paraganglioma of the urinary bladder: A lesion that may be misdiagnosed as urothelial carcinoma in transurethral resection specimens. Am J Pathol 2004;28:94-100.

18 Higgins JP, Kaygusuz G, Wang L, et al. Placental S100 (S100P) and GATA3: Markers for transitional epithelium and urothelial carcinoma discovered by complementary DNA microarray. Am J Surg Pathol 2007;31: 673-680.

19 Liu H, Wilkerson ML, Lin F. Immunhistochemical evaluation of GATA3 expression on tumors and normal tissues. A useful immunomarker for breast and urothelial carcinomas. Am J Clin Pathol 2012;138: 57-64.

20 Netto GJ, Epstein JI. Immunohistology of the prostate, bladder, kidney and testis, In Diagnostic Immunohistochemistry, Theranostic and Genomic Applications, 3rd edn. Saunders/Elsevier: Philadelphia, PA, USA; 2010, pp 593-661. 